\title{
Préservation de la fertilité chez les enfants et les adolescents traités pour un cancer. Aspects relationnels et éthiques
}

\author{
Daniel OPPENHEIM1, Laurence BRUGIERES2, Olivier HARTMANN2 \\ 1 Département de Pédiatrie et unité de psycho-oncologie \\ 2 Département de Pédiatrie. Institut Gustave Roussy, 94805 Villejuif
}

\section{RÉSUMÉ.}

La proposition faite à un adolescent de préserver sa fertilité avant une chimiothérapie qui peut provoquer sa stérilité soulève de nombreuses difficultés relationnelles et éthiques, parce qu'il s'agit d'un adolescent, de cancer, de sexualité, de parentalité. L'objectif est de préserver sa fertilité mais sans augmenter son désarroi et sa souffrance, sans provoquer un sentiment de culpabilité ou de perte irréparable en cas d'échec, sans gêner sa relation à ses soignants oncologues, sans altérer son estime de luimême et sa dynamique existentielle, sans que plus tard le souvenir de sa maladie et de son traitement reste honteux ou traumatique. En pratique courante, il importe d'être attentif à deux difficultés : en cas d'impossibilité ou d'échec du recueil (comment l'adolescent réagira ?), et quand les parents sont excessivement réticents, ou demandeurs à la place de l'adolescent. Dans ces situations, et encore plus quand est proposée une pratique rare et innovante, il importe de tenir compte des principaux repères d'éthique clinique : le respect de l'autonomie et de la dignité du patient, les principes de bienfaisance et de non malfaisance, le principe de justice. II importe aussi de s'interroger sur les objectifs, les attentes et les motivations de l'adolescent (qu'il accepte ou refuse la proposition) qui ne sont pas forcément les mêmes que ceux du médecin ou des parents. Le dialogue n'est pas toujours facile parce que le diagnostic de cancer (et le choc qui en découle) est récent, que le traitement doit débuter rapidement, que le médecin et l'adolescent se connaissent encore peu. Une formation au dialogue et à la relation, à la spécificité et à la psychologie de l'adolescent, à la résolution des difficultés éthiques dans le champ médical peuvent être utiles.

Mots clés : Adolescent adolescent, préservation de la fertilité, psychologie, éthique, relation soignant-patient

\section{INTRODUCTION}

La proposition de recueil de sperme chez un adolescent avant une chimiothérapie qui peut provoquer sa stérilité soulève de nombreuses difficultés relationnelles, éthiques, légales. [1]. Nous ne reprendrons pas les points que nous avons développés l'an dernier au colloque de la Fédération des CECOS en 2004 [5]. Nous développerons ici quelques unes des difficultés relationnelles et éthiques qui se retrouvent dans ces situations de proposition de recueil de sperme avant une chimiothérapie qui peut provoquer la stérilité. Ces situations sont complexes, parce qu'il s'agit de cancer, de traitement longs, éprouvants, qui comportent leurs effets adverses et leurs propres risques, parce qu'il s'agit d'adolescents [4] et enfin de sexualité, et pas simplement d'un acte visant à préserver la fertilité. C'est pourquoi il importe que cette proposition et ce geste n'augmentent pas le désarroi et la souffrance qui viennent du cancer [6], ne gênent pas sa relation à ses soignants oncologues, ne déstabilisent pas sa relation à lui-même (son estime de lui-même, sa dynamique existentielle) et aux adultes. II serait dommage que plus tard le souvenir de sa maladie et de son traitement soit traumatique et que l'adolescent assume mal ce qui aura été fait et la façon dont il se sera comporté pendant cette période de sa vie.

Bien souvent, la proposition est faite dans un contexte difficile : le diagnostic est encore récent, l'adolescent et ses parents sont encore sous le choc, dans un grand désarroi, pensant au pire, à la mort, ignorant quasiment tout du fonctionnement du service d'oncologie, des modalités et de la

\section{Correspondance}

Dr Daniel OPPENHEIM - Département de Pédiatrie et unité de psycho-oncologie. -

Tel 01.42.11.48.42 - Fax 01.42.11.52.75 -

E.mail : Openheim@igr.fr 
durée du traitement, de la façon dont ils pourront organiser leur vie pendant cette période. La relation aux soignants et au pédiatre oncologue est récente et fragile. De plus, ils doivent souvent d'emblée signer plusieurs consentements éclairés en rapport à des études cliniques de nature diverses. Certains parents, à cette occasion, se trouvent brutalement confrontés à la sexualité de leur enfant qu'ils avaient jusqu'alors pensé appartenir à un avenir encore lointain, ou qu'ils avaient plus ou moins volontairement ignorée. C'est dire que l'adolescent et ses parents sont peu réceptifs à la préoccupation de préserver la fertilité et qu'ils ont tendance à l'accepter, à la négliger ou la refuser sans véritable réflexion. Ainsi, c'est bien souvent le médecin qui doit porter pour eux cette préoccupation, avec une réflexion argumentée et la conviction qu'ils seront en accord avec la décision et l'assumeront dans un second temps. En cas d'impossibilité ou d'échec du recueil, le dialogue aide l'adolescent (et ses parents de leur côté) à exprimer leur point de vue et à trouver le juste équilibre entre la banalisation ( $\mathrm{Ce}$ n'est pas grave, l'important est le traitement et la guérison. "), les regrets excessifs ("Je ne pourrai jamais avoir d'enfants. " "Nous ne serons jamais grands parents. "), le sentiment de culpabilité (s'attribuant, en relation à des raisons physiques ou psychologiques la responsabilité de l'échec), l'analogie inadéquate ("C'est un signe de mauvais augure pour le traitement. »).

Les difficultés sont encore accentuées quand les méthodes proposées ne sont ni 'naturelles' ni de routine. II peut s'agir d'une méthode plus troublantes pour l'adolescent ou pour ses parents, parfois aussi pour son amie ou ses soignants, parce qu'elle nécessite anesthésie et intervention chirurgicale (biopsie testiculaire), avec leurs propres risques, parce qu'elle suscite chez certains une gêne ou un imaginaire excessif (stimulation endorectale). Dans de telles situations les questions éthiques cliniques se posent avec encore plus d'acuité [2]. Elles peuvent se formuler ainsi : «Ce que je fais respecte-t-il l'autonomie du patient, les principes de bienfaisance et de non malfaisance, de justice et de dignité ? " D'autres questions s'y ajoutent : "Qui est cet adolescent pour moi ? Qui suis je pour lui ? Quelle est la nature de notre relation ? Quelle est ma motivation? Qu'est ce qui m'autorise à faire ce que je lui fais ? Jusqu'où puis-je l'assumer ? " II est d'autant plus difficile, et nécessaire, de commencer à répondre à ces questions que la relation entre le pédiatre oncologue et l'adolescent est encore récente. Reprenons ces éléments.

\section{L'AUTONOMIE}

L'autonomie nécessite le libre jugement, la libre pensée, la libre décision. Elle dépend donc aussi de la bonne information [8], non seulement sur la valeur de l'objectif mais aussi sur celle de la méthode, avec ses avantages, ses limites, ses risques, et les éventuelles alternatives qui existent. L'information aide l'adolescent et ses parents à bien séparer l'intérêt de la proposition pour lui (les objectifs cliniques) et les enjeux d'une éventuelle recherche; à distinguer son intérêt, à court et à long terme ; son intérêt et celui éventuel de ses parents.
L'autonomie nécessite une sérénité de penser suffisante, qui n'est pas toujours présente dans ce contexte, bien souvent encore très proche du diagnostic et de la rencontre avec l'équipe d'oncologie. Mais compte tenu de l'importance de la question pour l'avenir (assumer sa parentalité, ou sa stérilité en cas d'insuccès), il est raisonnable de chercher autant que possible, ce qui n'est pas toujours facile, à disposer d'un temps suffisant de dialogue et de réflexion.

1. II importe que l'enfant ou l'adolescent, mais aussi ses parents, comprennent et assument ce qui est proposé et ce qui est fait. Ce n'est pas toujours facile, et pas seulement pour des raisons cognitives, socio-culturelles ou linguistiques. La logique et le raisonnement médical sont parfois fort éloignés de ceux des familles, les priorités ne sont pas toujours perçues de la même façon, l'émotion et le désarroi font obstacle à une bonne compréhension. C'est pourquoi la discussion ne doit pas être à sens unique mais être un authentique dialogue, évolutif. II ne suffit pas demander si la proposition à été comprise mais de vérifier dans le dialogue qu'elle l'a été véritablement et suffisamment.

2. Les adolescents, encore plus que les autres, ont besoin de se dire qu'ils ont pris ou accepté la décision en toute liberté, que la décision est bien la leur [9]. Mais la situation ne permet pas toujours que le processus décisionnel se déroule de façon satisfaisante. Le diagnostic de cancer est encore récent, l'adolescent, comme ses parents et/ou sa compagne, en est bouleversé, sidéré, il pense d'abord au risque vital, puis à toutes les contraintes et perturbations que la maladie et le traitement vont provoquer dans sa vie, ses activités, ses relations, ses projets. II se demande comment il pourra s'organiser pendant tout le temps du traitement. La préservation de la fertilité ne lui apparaît pas comme une priorité ("On verra ça plus tard" dit-ils souvent, de même que ses parents), et c'est le médecin qui doit souvent porter dans un premier temps cette préoccupation.

L'adolescent peut râler, protester, il importe de tenir bon car ces affrontements, ces plaintes et ces protestations, justifiés ou non, font partie du jeu relationnel nécessaire à l'adolescent. L'adolescent, pour soutenir sa confiance si fragile dans sa valeur et dans son chemin vers son indépendance, a besoin d'avoir en face de lui des interlocuteurs fermes et solides, respectueux aussi de ses hésitations et de ses doutes. Ils doivent avoir le sens de la négociation et du compromis mais aussi celui de leur responsabilité et des limites à ne pas dépasser, celles au delà desquelles les risques pour l'adolescent seraient excessifs ou la qualité de l'acte médical ne pourrait être préservée. Pour que le médecin puisse être cet interlocuteur solide et fiable face à l'adolescent, il est nécessaire que sa proposition s'appuie sur des arguments solides: sa compétence et celle de l'équipe soignante, l'équilibre entre le bénéficie que le patient peut en attendre et les risques ou les inconvénients qu'il subira, entre son intérêt et ceux des patients qui viendront après lui, quand il s'agit d'une méthode encore rare et innovante. Pour ces raisons, il importe de ne pas s'arrêter à ses premières expressions de réticence ou de refus. D'autant plus 
que l'adolescent est ambivalent et a tendance à osciller entre certitude et doute, confiance excessive et méfiance a priori, entre acceptation trop facile de la parole de l'autre et refus systématique de ce qui lui est dit, entre prudence et prise de risque, ouverture à l'autre et repli sur soi, optimisme et pessimisme, sentiment de la continuité et anticipation et tentation de vivre au jour le jour, entre liberté de l'improvisation et réassurance de repères solides, entre sentiment de la différence (de son identité unique) et désir que rien ne le distingue des autres, besoin du groupe et besoin équivalent de sa famille, etc.

3. Sa révolte ou son comportement 'insupportable' envers ses parents ou ses soignants témoignent souvent de sa crainte d'être passif, position insupportable à ses yeux. II 'en fait trop' pour bien montrer et se prouver qu'il n'est pas passif, qu'il n'est pas tenté de l'être. Or, dans sa situation de patient, tout convergera vers cette position de passivité : la maladie et le traitement qui entraînent fatigue et douleur, dépendance envers les soins que prodiguent les infirmières et le savoir que possèdent les médecins (il sait que sa guérison dépend d'eux, pas de sa volonté). Les traitements qu'il recevra, parfois éprouvants, lui feront souhaiter ou demander la présence de ses parents (et c'est surtout sa mère qui sera disponible), et risqueront d'accentuer ce sentiment de retour à la dépendance de l'enfance, dont il a eu parfois (et ses parents avec lui) tant de mal à se déprendre.

4. L'adolescent, mais l'enfant aussi, a besoin de connaître l'ensemble des considérations de la proposition qui lui est faite : la raison qui en est à l'origine et qui la guide (dans quelle logique elle s'intègre, pas seulement le fait qu'elle est jugée nécessaire par le médecin et par ses parents), les objectifs (qui ne sont pas forcément les mêmes que les siens), les risques et les effets secondaires, mais aussi les conséquences, aussi bien dans le cas où il accepte la proposition que dans celui où il la refuse (et donc le risque éventuel, et sa gravité, qu'il prend en refusant).

II n'est pas toujours facile pour le médecin de laisser la discussion prendre le temps qui lui est nécessaire : celui dont il dispose est parfois limité, la maladie a parfois ses propres exigences et le traitement doit démarrer rapidement. Pourtant, une décision authentiquement assumée, constitue la meilleure garantie pour que plus tard, quelle que soit l'issue, ce qui aura été décidé et fait ne devienne pas la cause de regrets répétitifs, d'amertume, de perte d'estime de soi-même, de souffrance. Dans le souvenir global qu'il gardera de cette période de sa vie, tous les éléments qui auront participé à l'expérience qu'il aura traversée joueront leur rôle - qu'ils aient été médicaux ou relationnels, importants ou secondaires du point de vue médical - et feront qu'il sera à l'aise avec sa vie ou encombré par le souvenir qu'il gardera. Si l'adolescent meurt, quel souvenir les parents garderont de lui, et de leur responsabilité envers lui (avoir été responsables d'une souffrance supplémentaire, et en fin de compte inutile ?) ; s'il guérit, quel regard rétrospectif portera-t-il sur cette période importante de sa vie ?. En garde- ra-t-il le sentiment d'avoir été passif, d'avoir tout accepté sans comprendre, même s'il reconnaît que c'était pour son bien, ou au contraire d'avoir été à la hauteur (non pas 'courageux' mais d'avoir été partie prenante), d'avoir cherché à comprendre, à donner son point de vue, à rester lui-même, de s'être adapté à une situation inhabituelle, troublante et complexe, et le recueil de sperme est un des premiers éléments de cette épreuve, de cette expérience ? Une telle décision est le résultat d'un dialogue et du cheminement de sa propre réflexion : il ne suffit pas d'être convaincu par les arguments de l'interlocuteur, il lui est nécessaire aussi d'avoir fait le tour des siens, d'avoir repris à son compte ceux de l'autre après les avoir évalués et contestés.

5. Dans l'expérience que fait l'adolescent du traitement de son cancer, il n'y a pas de grandes et petites questions, toutes sont liées entre elles. II peut accorder une extrême importance à des éléments que le médecin et les parents jugent mineurs et secondaires du point de vue médical. Mais, négligés par le médecin, ils pourront devenir le point d'appui ou de cristallisation d'un rejet plus global et dangereux de l'ensemble du traitement. Des adolescents ont pu s'engager dans un bras de fer dramatique (en refusant par exemple telle chimiothérapie, pour une raison en apparence dérisoire, sachant bien les risques majeurs qu'ainsi ils couraient) avec les médecins, mais cet affrontement absurde cachait en fait une relation perturbée à leurs parents. Leur attitude apparente cachait un enjeu profond, véritablement vital, qui pouvait, par exemple, s'exprimer ainsi : " Jusqu'où tenez vous à moi ? Jusqu'où êtes vous prêts à respecter mon autonomie et ma dignité ? Jusqu'où êtes vous prêts à assumer votre autorité et votre responsabilité de parents?"

6. Le désarroi de l'adolescent a de multiples causes. La nouveauté de sa situation bien souvent (il ne connaît ni cette maladie, ni l'hôpital et ses façons de faire et de penser), l'absence de ses interlocuteurs habituels, qu'il s'agisse de ses parents (souvent eux-mêmes trop bouleversés dans un premier temps pour occuper cette place), de ses copains (qui n'en savent pas plus que lui sur les meilleures façons de se comporter dans cette situation nouvelle, de s'y confronter, qui ne viennent pas facilement à l'hôpital, et lui-même n'a pas forcément envie de se montrer à eux sous une image dégradée qui risquerait de lui 'coller durablement à la peau').

7. Les questions de la mort et de la parentalité sont souvent liées chez l'adolescent. La mort le renvoie à celle de son corps, de ses désirs, de son identité d'enfance, mais aussi à celle, advenue ou à venir, de ses parents et de ses grands parents. La quête de l'autonomie et de la maturité qui le caractérise s'associe au vieillissement inévitable de ses parents. Ceux-ci l'acceptent parfois mal, surtout si cet adolescent malade est leur dernier enfant, et ils savent qu'ils n'en auront plus d'autre, que l'étape suivante pour eux est la retraite et la vieillesse. La proposition de préservation de la fertilité les projette brutalement dans cet avenir, plus ou moins proche, et il leur est difficile d'éviter d'y penser, ce qui 
introduit une préoccupation parfois désagréable et qui les décentre de celle de la maladie, qu'il voudrait exclusive. Certains adolescents sont au seuil de l'âge adulte et de ses préoccupations (pour eux-mêmes et pour leurs parents) et d'autres sont encore très proches de l'enfance ; leurs affects et leurs pensées, leur questionnement ne sont pas les mêmes, et leurs réactions face à la proposition et au résultat de sa réalisation (succès ou insuccès) sont différents : les uns peuvent en être paradoxalement soulagés, les autres attristés.

8. L'attitude de l'adolescent est différente selon qu'il vient d'entrer dans le processus de l'adolescence ou qu'il est proche de l'adulte (il s'agit autant de critère d'âge que de maturité). Les uns sont encore très dépendants de leur identification au père (est-il un modèle paternel satisfaisant, faut-il chercher à lui ressembler ou au contraire prendre son contre-pied) et de leur attachement à la mère (comment acceptera-t-elle la perspective du départ de son fils, comment réagira-t-elle quand il prendra son indépendance d'adulte, et ils perçoivent que ces questions sont liées mais cette perspective la renvoie aussi à la crainte de leur mortle perdre). Si ces questions que suscite chez les parents et chez l'adolescent la proposition de prélèvement de sperme - et qui ne sont pas toujours clairement formulées ni conscientes - ne sont pas prises en compte par le médecin, elles risqueront d'accentuer leur trouble, qui de plus ne sera que partiellement compris et insuffisamment aidé.

9. L'adolescent est aussi particulièrement attentif à la façon dont ses parents se comportent dans cette situation : sont-ils à la hauteur, peut-il compter sur eux, en être fier? D'où l'importance de ne pas tomber dans le piège que tend l'adolescent aux soignants quand il critique ses parents, ni quand il en fait un éloge excessif, ou quand il dit, aux uns ou aux autres : "C'est vous qui savez, je vous fais confiance, je vous laisse décider. " Pour cette raison, il est souhaitable de discuter aussi avec les parents, avec l'accord de l'adolescent, y compris quand il est mineur.

10. L'accord des parents à la préservation de la fertilité de leur enfant masque parfois une certaine ambivalence : ils souhaitent bien sûr qu'il devienne père un jour (surtout si la naissance d'un garçon leur permet de voir leur nom se perpétuer) et ils seront très contents de devenir grands parents, mais cette perspective d'avenir les renvoie aussi à leur vieillissement. Cette ambivalence explique certaines de leurs attitudes paradoxales [7], quand ils disent : "Ce n'est pas la priorité, on verra ça plus tard, il a le temps, il pourra toujours adopter un enfant, etc. ", phrases que l'adolescent peut reprendre à son compte pour ne pas les troubler plus ou s'opposer à eux, parce qu'il sait qu'il a besoin d'eux pour la traversée de son traitement.

\section{LA BIENFAISANCE}

L'objectif premier de l'acte est d'apporter à l'adolescent un bénéfice, et largement supérieur aux inconvénients ou aux risques. II importe donc qu'il soit convaincu de l'intérêt de l'enjeu, même s'il ne le perçoit pas encore toujours clairement, d'autant qu'il n'en bénéficiera pas au temps présent mais dans un avenir plus ou moins lointain.

\section{LA NON MALFAISANCE}

Eviter de lui faire ce qui pourrait lui nuire, lui faire du mal, qu'il s'agisse de son corps, de ses relations amicales ou affectives, de son état psychologique, de sa situation sociale ou familiale, etc. Aucun acte n'est anodin, et il importe de bien en peser les avantages et les inconvénients pour lui.

\section{LA JUSTICE}

Que ce qui fait proposer ou ne pas proposer tel acte à l'adolescent ne découle pas de raisons autres que celles de son intérêt et des nécessités ou des possibilités médicales. La discrimination pour des raisons raciales, religieuses, économiques et sociales n'a bien sûr pas sa place dans le champ médical. Mais il peut s'agir de formes plus subtiles, par exemple attribuer à l'adolescent ses propres hésitations ou les réticences et les doutes des parents.

\section{LA DIGNITÉ}

Que ni sur le moment ni rétrospectivement dans l'aprèscoup l'adolescent ne pense avoir été utilisé pour des enjeux qui n'étaient pas forcément les siens, instrumentalisé, manipulé, y compris avec sa complicité ; qu'il n'ait pas le sentiment que ce qui lui a été fait a été dégradant, qu'il en ait honte, qu'il ne puisse l'intégrer dans l'image qu'il a de luimême, qu'il ne puisse le raconter sans gêne psychique et morale excessives à ceux en qui il a confiance; que le malaise ou la révolte qu'il en éprouve s'étendent en tache d'huile à l'ensemble du traitement qu'il aura eu et lui rendent sa guérison insupportable.

\section{LA QUESTION DE LA NATURE DE LA RELATION ENTRE LUI ET LE MEDECIN}

La question de la nature de la relation entre lui et le médecin en est, pour ces raisons, cruciale, au delà de l'évidence des soins.

"Qui est-il pour moi ? Qui suis-je pour lui ? Que me veuti) ? Qu'est ce qui l'autorise à me faire ce qu'il me fait ? Que veut-il pour moi ? Qu'est ce que j'attends de lui ? " sont des questions fréquentes dans ces situations, mais pas toujours clairement formulées. L'adolescent, encore plus que l'enfant ou l'adulte, en raison de l'instabilité et du doute qui sont constitutifs de la période même de transition que représente l'adolescence, se demande quel est le désir de l'adulte, et s'il peut lui faire confiance. Dans ce contexte, il se demande aussi de qui est le désir de ce qui lui est fait : du médecin, de ses parents, le sien propre. 
L'adolescent attend beaucoup du médecin, non seulement l'efficacité thérapeutique mais aussi l'intérêt, l'estime, le respect, l'affection, et celui-ci est parfois mis par lui dans la position ou l'image de l'adulte qui remplace un parent ou un grand parent. Inversement, l'adolescent parfois, à son insu, renvoie le médecin à des personnes qui ont compté, qui continuent de compter pour celui-ci. Le médecin alors peut revivre, sans toujours en être conscient, des situations qu'il a vécues dans sa vie personnelle ou professionnelle. Il est souhaitable que le médecin soit, dans un tel contexte chargé d'émotion intense et d'enjeux importants, un minimum conscient des raisons mêmes de sa vocation, qu'elle soit celle de médecin, d'oncologue, de praticien des CECOS, etc., car ces raison mêmes se réactualisent dans chaque acte et particulièrement dans ceux qui posent des problèmes relationnels ou éthiques complexes. De même il est bon que le médecin croie aux enjeux de ce qu'il propose, à son utilité pour le patient mais aussi aux progrès que l'expérience acquise avec chacun feront faire à la médecine, aux bénéfices qu'ils apporteront aux autres patients, mais toujours avec un certain recul pour que son enthousiasme ou son désabusement ne lui fassent pas sous ou surestimer la réticence et les désagréments ou les bénéfices de l'acte pour l'adolescent.

\section{EN CONCLUSION}

L'essai de préserver la fertilité d'un adolescent traité pour une maladie grave a une double importance : pour lui-même et pour la place qu'il occupe dans le traitement global de cette maladie. II n'est pas un acte technique, simple ou complexe, dont la justification médicale apparaît évidente. II a des conséquences sur la relation de l'adolescent à sa maladie et à son traitement, à lui-même, aux adultes, et d'abord à ses parents, et à la médecine. Il engage le présent mais aussi l'avenir et le devenir à long terme de cet adolescent.

II peut poser des questions relationnelles et éthiques complexes, dans les pratiques cliniques de routine et encore plus dans les pratiques rares et innovantes, auxquelles il importe de rester attentif et de se donner les moyens d'y répondre. Un travail d'équipe, auquel le psychanalyste participe, une formation au dialogue et à la relation, à la spécificité et à la psychologie de l'adolescent, à la résolution des difficultés éthiques dans le champ médical peuvent être utiles, ainsi que l'établissement de recommandations et de bonnes pratiques [3].

\section{REFERENCES}

1. BAHADUR G., WHELAN J., RALPH D., HINDMARSH P. : Gaining consent to freeze spermatozoa from adolescents with cancer ; legal, ethical and practical aspects. Hum. Reprod., 2001, 16 : 188-193.

2. FAGOT-LARGEAULT A. : L'éthique. In : Caulin C., Chastang C., Dahan R. eds. Méthodologie de l'évaluation thérapeutique. Paris, Masson, 1993 : 57-74.
3. GLASER A., WILKEY O., GREENBERG M. : Sperm and ova conservation : existing standards of practice in North America. Med. Ped. Oncol., , 2000, 35 : 114-118.

4. JEAMMET P. : Adolescence et processus de changement., in : Widlöcher D. ed. Traité de psychopathologie. Paris, P.U.F., 1994 : 687-726.

5. OPPENHEIM D, BRUGIERES L, HARTMANN O. : Préserver la fertilité chez les adolescents traités pour un cancer. Le point de vue du psycho-oncologue. Andrologie, 2004, 14 : 422-427.

6. OPPENHEIM D. : Grandir avec un cancer. L'expérience vécue par l'enfant et l'adolescent. Bruxelles, De Boeck, 2003.

7. SCHOVER R.L. : Psychosocial aspects of infertility and decisions about reproduction in young cancer survivors : a review. Med. Ped. Oncol., 1999, $33:$ 53-59.

8. SCHOVER L.R., RYBICKI L.A., MARTIN E.A., BRINGELSEN K.A. : Having children after cancer. Cancer,. 1999, 86 : 697709.

9. SCHOVER R.L., BREY K., LICHTIN A., LIPSCHULTZ L.I., , JEHAS. : Knowledge and experience regarding cancer, infertility, and sperm banking in younger male survivors. J. Clin. Oncol., 2002, 7 : 1880-1889.

Communication au XIII Séminaire de la Fédération Française des CECOS, Tours, 7-9 Avril 2005.

Manuscrit reçu : avril 2005 ; accepté mai 2005.

\section{ABSTRACT}

Fertility preservation in male childboys and adolescents treated for cancer patients. Relational and ethical issues.

\section{Daniel OPPENHEIM, Laurence BRUGIERES, Olivier HARTMANN}

Proposing fertility preservation to an adolescent before chemotherapy that may induce sterility raises numerous relational and ethical issues, as it concerns an adolescent, cancer, sexuality, and parenthood. The goal is to preserve fertility without exacerbating confusion and suffering, without triggering a sense of guilt or irretrievable loss in the event of failure, without hampering his relations with the oncologist caregiver, without transforming his self-esteem and existential dynamics, so that later the memory of the illness and its treatment will not be shameful or traumatic.

In routine practice, it is important to be attentive to two problems: if semen collection is impossible or fails (how will the adolescent react?) and when parents are too reticent or too eager to do so in the adolescent's place. In these situations, and even more so when a rare, innovative procedure is proposed, it is important 
to take into account the main principles of clinical ethics: respect the patient's autonomy and dignity, the principles of causing no harm and justice. It is also important to determine the adolescent's objectives, expectations and motivations (whether he accepts or refuses the proposal) which are not necessarily the same as those of the clinicians or parents.

Dialogue is not always easy because the diagnosis of cancer (and the resulting shock) is recent, the treatment must commence rapidly, and the doctor and patient do not know each other very well. Training in the use of dialogue and relational skills, in issues specific to adolescents and adolescent psychology, and in solving ethical problems in the medical field can be useful.

Key words: adolescent, fertility preservation, psychology, ethics, care provider-patient relationship 